\title{
Midkine: Utility as a Predictor of Early Diabetic Nephropathy in Children with Type 1 Diabetes Mellitus
}

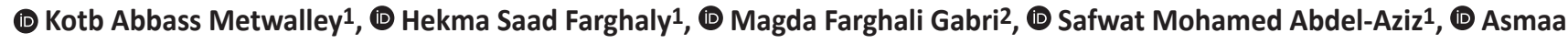 \\ Mohamed Ismail2, (1) Duaa Mohamed Raafat ${ }^{1}$, (D) Islam Fathy Elnakeeb3
}

${ }^{1}$ Assiut University Faculty of Medicine, Department of Pediatrics, Assiut, Egypt

2Aswan University Faculty of Medicine, Department of Pediatrics, Aswan, Egypt

${ }^{3}$ Aswan University Faculty of Medicine, Department of Clinical Pathology, Aswan, Egypt

\section{What is already known on this topic?}

Microalbuminuria is the gold standard for the detection and prediction of diabetic nephropathy (DN). However several studies have indicated that microalbuminuria lacks specificity for accurate prediction of DN.

\section{What this study adds?}

Serum midkine is a useful, novel, practical marker for the evaluation of renal involvement in children with type 1 diabetes mellitus, especially in normoalbuminuric children.

\begin{abstract}
Objective: This study aimed to assess the role of serum midkine (MK) as a biomarker for early detection of diabetic nephropathy in children with type 1 diabetes mellitus (T1DM) before microalbuminuria emerges.

Methods: A total of 120 children with T1 DM, comprising 60 microalbuminuric patients (Group 1), 60 normoalbuminuric patients (Group 2), and 60 healthy participants as a control group (Group 3) were included. Detailed medical history, clinical examination, and laboratory assessment of high-sensitivity C-reactive protein (hs-CRP), hemoglobin A1c percentage (HbA1c\%), lipid profile, urinary albumin to creatinine ratio (ACR), serum MK and estimated glomerular filtration rate based on serum creatinine were performed in all participants. Results: Both Group 1 and Group 2 had significantly higher serum MK compared to controls ( $<<0.001)$. Additionally, significantly higher MK concentrations were present in Group 1 compared with Group $2(p<0.001)$. Receiver operating characteristic curve analysis revealed that the MK concentration cutoff value of $1512 \mathrm{pg} / \mathrm{mL}$ was able to predict microalbuminuria with a sensitivity of $96 \%$ and specificity of $92 \%$. Stepwise regression analysis revealed that HbA1c\%, hs-CRP, and ACR were independently related to MK levels ( $p<0.001$ for each). Conclusion: The results of this study suggest that serum MK is a useful, novel, practical marker for the evaluation of renal involvement in children with T1DM, especially in normoalbuminuric children.
\end{abstract}

Keywords: Midkine, type 1 diabetes mellitus, diabetic nephropathy, urinary albumin creatinine ratio

\section{Introduction}

Midkine (MK) is a multifunctional heparin-binding growth factor that was primarily identified as the retinoic acidresponse gene product (1). It has pleiotropic activities including enhancement of cell proliferation, differentiation, survival, and migration and is also involved in angiogenesis and oncogenesis $(2,3)$. Furthermore, functional evidence has suggested a possible role for MK in modifying inflammatory responses (4). MK is implicated in the pathogenesis of multiple disease processes, including cancer development, neuronal survival, tissue inflammation, and acute and chronic kidney disease (CKD) $(5,6,7)$. In the kidney, MK is expressed in both proximal tubular cells and distal tubular epithelial cells (7) and to a lesser extent in endothelial cells (8). MK is induced by oxidative stress via the activation of 
hypoxia-inducible factor-1-alpha (7). The pathological roles of $\mathrm{MK}$ in renal disease are broad, ranging from progression of CKD (8), to hypertension (9), diabetes mellitus (DM) associated kidney damage (10) and drug toxicity (11).

Diabetic nephropathy (DN) is a grave complication that may occur in both type 1 and 2 DM and, unless arrested, leads to end-stage kidney disease (12). Pathologically, DN is a diffuse process affecting glomerular endothelial cells, tubular epithelial cells, and interstitium (13). The natural history of DN may be divided into several stages beginning with glomerular hyperfiltration and progressing to the silent phase (normoalbuminuria), incipient nephropathy (microalbuminuria), overt nephropathy (macroalbuminuria), and finally established renal failure $(14,15)$. The occurrence of glomerular basement membrane and tubular basement membrane thickening on histopathology in type 1 diabetes mellitus (T1DM) kidney tissues suggests that tubular injury is not independent of glomerular injury, as both can occur after a same duration of disease (16). As with other kidney diseases, the outcome of diabetic kidney disease is better determined by tubulointerstitial changes than glomerular changes (17). Classically, microalbuminuria is the gold standard for the detection and prediction of DN (12). However, studies have shown that histopathologic changes associated with DN may occur in normoalbuminuric diabetic patients (18). Furthermore, microalbuminuria appears once significant renal damage has actually occurred (19). Moreover, there are several confounding factors with which microalbuminuria is associated, such as urinary tract infection, or following exercise or acute illness, thus indicating the non-specificity of the presence of microalbuminuria for accurate prediction of DN (20). Consequently, there is a urgent need for a more specific and sensitive biomarker for earlier diagnosis of DN during the "tubular stage" of renal damage, before microalbuminuria appears. To the best of our knowledge, there are no data available regarding the association between serum MK and DN in children with T1DM. The current study aimed to assess the diagnostic value of serum MK as a novel biomarker in the prediction of microalbuminuria, thus allowing for early recognition of DN in children with T1DM before the manifestation of microalbuminuria was evident.

\section{Methods}

\section{Patients}

This is a case-control study included sixty children and adolescents with T1DM having microalbuminuria, defined as urinary albumin excretion 30-299 mg/g creatinine, (the microalbuminuric group; Group 1) and sixty children and adolescents with T1DM who were normoalbuminuric, defined as urinary albumin excretion $<30 \mathrm{mg} / \mathrm{g}$ creatinine, (the normoalbuminuric group; Group 2) (21). Exclusion criteria were: the presence of any clinical or laboratory evidence of chronic infection, immunosuppression, liver diseases, heparin therapy, connective tissue disease, or other autoimmune disorders. Patients on antiplatelet drugs, lipid-lowering medication, or anti-hypertensive therapy including angiotensin-converting enzyme inhibitors (ACEIS) or angiotensin receptor blockers (ARBs) were also excluded, as the protective role of both ACEIs and ARBs on glomerular and tubulointerstitial compartments have been proven in human studies (22). Patients were recruited over a period of two years, from April 2017 to March 2019, from the outpatient pediatric diabetes clinic of the Children's Hospital, Assiut University, Assiut, Egypt. This study also included sixty healthy children and adolescents who were recruited from the general population and matched for age, sex, pubertal stage, body mass index (BMI) standard deviation (SD) score (SDS), and socioeconomic status; these healthy children were designated Group 3. The study protocol was approved by the Ethics Committee of the Faculty of Medicine, Assiut Children's University Hospital, Assiut, Egypt (21/2017). Informed consent was obtained from each patient or control subject or their legal guardians before enrollment into the study.

\section{Methodology}

A detailed medical history was obtained from the studied patients were also subjected to a thorough clinical examination, with special emphasis on the age of onset of diabetes, disease duration, and insulin dose. Anthropometric measurements including weight, height, and waist circumference were obtained by a trained nurse according to standardized techniques. BMI was calculated as weight in kilograms divided by squared height in metres $\left(\mathrm{kg} / \mathrm{m}^{2}\right)$. These BMI values were then converted to SDS using reference data for Egyptian children and adolescents (23). Puberty was assessed using the standardized method of Tanner stages (24). Systolic and diastolic blood pressures (SBP and DBP, respectively) were measured by standard technique. SDS for mean BP were calculated according to the report of the National High Blood Pressure Education Program Working Group on High Blood Pressure in Children and Adolescents (25). Hypertension was defined as mean systolic or mean diastolic BP >1,645 SDS as 1,645 SDS corresponds to the $95^{\text {th }}$ percentile in a standard normal distribution). 


\section{Laboratory Investigations}

Fasting lipid profile and high sensitivity C-reactive protein (hs-CRP) were measured using Cobas Integra 800 (Roche Diagnostics, Mannheim, Germany). Serum total cholesterol, high-density lipoprotein (HDL), and triglyceride concentrations were measured by standard enzymatic methods using commercial reagents (Boehringer Mannheim GmbH, Germany). Calculation of low-density lipoprotein (LDL) concentration was performed using Friedewald's equation (26). Assessment of mean hemoglobin A1C percentage (HbA1c\%) in the year preceding the study was performed using high-performance liquid chromatography on a Variant Analyzer (Bio-Rad Inc., Cairo, Egypt). Urinary albumin excretion (as an indicator of nephropathy) was measured in an early morning urine sample as an albuminto-creatinine ratio (ACR) by an immuno-nephelometric method on a prime photometer (BCP BioSed, Rome, Italy). Microalbuminuria is present if urinary albumin excretion in at least two out of three consecutive urine samples, two months apart was 30-299 mg/g creatinine and patients were defined as normoalbuminuric if urinary albumin excretion was $<30 \mathrm{mg} / \mathrm{g}$ creatinine (21). Potential factors affecting urinary albumin excretion, such as exercise, fever, and posture were excluded. Serum creatinine $(\mathrm{Cr}, \mathrm{mg} / \mathrm{dL})$ was measured on a Dimension Xpand plus chemistry analyzer using commmercial methods (Siemens Technology, Illinois, USA). Serum creatinine-based estimated glomerular filtration rate (eGFR-Cr) was calculated using the updated Schwartz formula. eGFR- $\mathrm{Cr}=0.413$ *height $(\mathrm{cm}) /$ serum $\mathrm{Cr}$ (mg/dL) (27). Determination of serum MK was performed using an enzyme-linked immunosorbent method (Human Midkine ELISA Kit, Boster Biological Technology Co., Pleasanton, CA, USA).

\section{Statistical Analysis}

Data were analyzed using Statistical Package for the Social Sciences (SPSS), version 19.0 (SPSS Inc., Chicago, IL, USA). Qualitative variables are presented as number and percent [n (\%)] and compared by the chi-square test. Quantitative variables were tested for normality using the KolmogorovSmirnov test. Normally distributed quantitative variables were expressed as mean and SD (mean \pm SD) and the oneway analysis of variance (ANOVA) test was used to compare the three studied groups with Bonferroni post-hoc test used to detect pair-wise comparison. Spearman correlation was used for non-parametric correlation between quantitative variables and Pearson correlation was used for parametric correlation. Multiple linear regression analysis was employed to assess the relationship between MK and clinical and laboratory variables. Logistic regression was used to examine the relationship between MK and ACR, after adjustment for other variables. Receiver operating characteristic (ROC) curve was used to determine the best cutoff value of MK in the prediction of microalbuminuria in children with T1DM. The area under the curve (AUC), specificity and sensitivity were computed based on the ROC. A p value $<0.05$ was considered significant in all analyses.

\section{Results}

The demographic data and laboratory finding of the patient groups and the control group are shown in Table 1. Children with T1DM, both Group 1 and Group 2, had significantly higher SBP SDS, DBP SDS, serum total cholesterol, LDL cholesterol, triglycerides, HbA1c\%, hs-CRP, ACR, and MK compared with control subjects $(p<0.05$ for all). Children in Group 1 were older with longer disease duration. They had significantly higher blood pressure SDS, HbA1 c, hs-CRP, urinary ACR, serum lipids (except HDL cholesterol), and insulin dose compared with Group 2 ( $p<0.05)$. ROC curve analysis revealed that the MK concentration cutoff value of $1512 \mathrm{pg} / \mathrm{mL}$ was able to distinguish microalbuminuria with a sensitivity of $96 \%$ and specificity of $92 \%$ [AUC, 0.94; confidence interval $(\mathrm{CI}), 0.87-1.00 ; \mathrm{p}<0.001]$ (Figure 1).

MK was positively correlated with disease duration, SBP and DBP SDS, HbA1c, hs-CRP, and urinary ACR $(p<0.05)$, while no correlation was found between $M K$ and age, serum lipids, BMI SDS, or insulin dose $(p>0.05)$ (Table 2). Stepwise regression analysis (Table 3) revealed that $\mathrm{HbA} 1 \mathrm{c}$, hs-CRP, and urinary ACR were independently related to MK levels $(p<0.001)$. Moreover, logistic regression revealed that MK was a significant independent factor for DN, after adjustment of other variables, including age, gender, disease duration, BMI SDS, BP, HbA1c, ACR, and fasting lipids (Odds ratio: $2.05,95 \% \mathrm{CI}: 1.16-5.26 ; \mathrm{p}<0.001)$.

\section{Discussion}

In this study, MK levels were found to be significantly higher in diabetic children with microalbuminuria compared to those with normoalbuminuria and controls so that in Group 1 the mean MK level was 1.6-fold higher than in Group 2 and 2.8-fold higher than in healthy children. Most importantly, the normoalbuminuric children had significantly higher levels of MK compared with controls (mean value 1.76fold higher). This suggests that serum MK levels are related to subclinical tubular impairment and may be used as an earlier, measurable marker of renal involvement before the onset of microalbuminuria. Furthermore, MK correlated positively and significantly with urinary ACR $(p<0.001)$, suggesting that MK may influence the severity of renal involvement and thus MK may be used as a marker to 
Table 1. Clinical and laboratory variables of diabetic patients (normoalbuminuria and microalbuminuria), and healthy control groups

\begin{tabular}{|c|c|c|c|c|}
\hline & $\begin{array}{l}\text { Microalbuminuric } \\
\text { (Group 1) }(n=60)\end{array}$ & $\begin{array}{l}\text { Normalbuminuric } \\
\text { (Group 2) }(n=60)\end{array}$ & $\begin{array}{l}\text { Healthy controls } \\
\text { (Group 3) }(n=60)\end{array}$ & $\mathrm{p}$ value \\
\hline Age & $16.5 \pm 2.7$ & $13.8 \pm 3.4$ & $14.3 \pm 3.9$ & 0.011 \\
\hline Male & $32(53.3)$ & $29(48.3)$ & $28(46.6)$ & 0.865 \\
\hline Diabetic duration (yrs) & $9.3 \pm 2.6^{\#}$ & $6.2 \pm 1.4$ & - & $<0.001$ \\
\hline Insulin dose (IU/kg/day) & $0.99 \pm 0.26$ & $0.89 \pm 0.27$ & - & 0.910 \\
\hline BMI SDS & $0.68 \pm 0.8$ & $0.64 \pm 0.4$ & $0.63 \pm 0.5$ & 0.172 \\
\hline SBP SDS & $1.77 \pm 0.4^{* \#}$ & $1.3 \pm 0.2^{*}$ & $0.63 \pm 0.2$ & $<0.001$ \\
\hline DBP SDS & $0.99 \pm 0.3^{* \#}$ & $0.4 \pm 0.1^{*}$ & $0.32 \pm 0.05$ & $<0.001$ \\
\hline Triglycerides (mg/dL) & $183.9 \pm 35.8^{* \#}$ & $126.7 \pm 16.9^{*}$ & $107.1 \pm 11.2$ & $<0.001$ \\
\hline Total cholesterol (mg/dL) & $198.7 \pm 37.3^{* \#}$ & $165.3 \pm 25.1^{*}$ & $127.3 \pm 15.3$ & $<0.001$ \\
\hline LDL cholesterol (mg/dL) & $136 \pm 35.4^{\# \#}$ & $94.7 \pm 22.5^{*}$ & $87.7 \pm 12.2$ & $<0.001$ \\
\hline HDL cholesterol (mg/dL) & $41.2 \pm 15.2^{* \#}$ & $61.7 \pm 15.9$ & $69.8 \pm 13.2$ & $<0.001$ \\
\hline ACR (mg/g creatinine) & $218.6 \pm 35.6^{* \#}$ & $22.2 \pm 2.7^{*}$ & $10.3 \pm 2.7$ & $<0.001$ \\
\hline $\mathrm{Hb} 1 \mathrm{Ac}(\%)$ & $9.2 \pm 1.3^{* \#}$ & $7.8 \pm 0.4^{*}$ & $4.7 \pm 0.3$ & $<0.001$ \\
\hline hs-CRP (mg/L) & $6.55 \pm 1.3^{* \#}$ & $2.81 \pm 0.78^{*}$ & $0.39 \pm 0.11$ & $<0.001$ \\
\hline Serum creatinine (mg/dL) & $0.71 \pm 0.11$ & $0.67 \pm 0.12$ & $0.65 \pm 0.13$ & 0.148 \\
\hline eGFR-Cr $\left(\mathrm{mL} / \mathrm{min} / 1.73 \mathrm{~m}^{2}\right)$ & $100.71 \pm 27.88$ & $103.09 \pm 33.22$ & $113.93 \pm 30.21$ & 0.208 \\
\hline Serum Midkine (pg/mL) & $1847.2 \pm 266.4^{* \#}$ & $1158.4 \pm 157.6^{*}$ & $658.3 \pm 79.3$ & $<0.001$ \\
\hline
\end{tabular}

Data are shown as mean \pm standard deviation, or $\mathrm{n}(\%)$.

"Indicates significance versus control subjects; \#indicates significance between Group 1 and Group 2.

yrs: years, BMI: body mass index, SDS: standard deviation score, LDL: low-density lipoprotein, HDL: high-density lipoprotein, ACR: urinary albumin creatinine ratio, HbA1 c: hemoglobin A1c, hs-CRP: high-sensitivity C-reactive protein, eGFR: estimated glomerular filtration rate, SBP: systonic blood pressure, DBP:

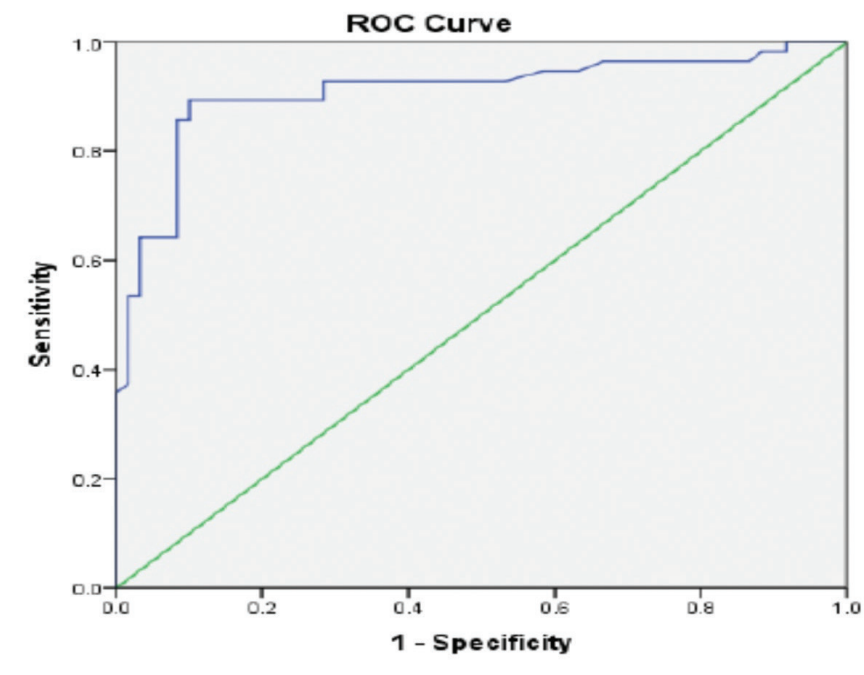

Figure 1. Receiver operating characteristic curve of fasting C-peptide to predict microalbuminuria with a sensitivity of $96 \%$ and specificity of $92 \%$ (area under the curve was 0.94)

stratify DN into different stages. In a previous study, Kosugi et al (10) reported that kidney biopsy tissue from eight adult patients with DN revealed that marked tubular atrophy, interstitial fibrosis and interstitial cell infiltration were evident in the specimens of DN, in which MK induction was detected in the glomeruli, tubules and interstitium. In addition, MK expression was detected in all the examined cases, despite the patients exhibiting different degrees of DN. These data were in agreement with the MK expression pattern in a mouse model induced by streptozotocin (10). Although glomerular dysfunction is thought to be a major factor in the development and progression of DN, tubulointerstitial damage may also play an important role in the pathogenesis of DN. MK is up-regulated in damaged tubular epithelial cells during the extremely early phase, both in vivo and in vitro, when only ischemia and hypoxia are evident (28). Experimental studies have shown that MK antisense oligodeoxynucleotides (anti-MK ODN) can improve ischemic reperfusion-induced renal damage, arterial restenosis and cisplatin-induced nephropathy (11). In line with our results, we suggest that MK inhibitors may be useful in treating DN, which may offer new avenues for the development of therapy for DN (29).

The International Society of Nephrology recommend annual screening for albuminuria and measurement of eGFR to detect and monitor DN in patients with DM (30). In the current study, no significant difference in eGFR-Cr among 


\begin{tabular}{|c|c|c|}
\hline Variable & $\mathrm{r}$ & $\mathrm{p}$ \\
\hline Age (yrs) & 0.298 & 0.071 \\
\hline Disease duration (yrs) & 0.411 & 0.006 \\
\hline BMI SDS & -0.086 & 0.514 \\
\hline SBP SDS & 0.465 & 0.002 \\
\hline DBP SDS & 0.311 & 0.002 \\
\hline $\operatorname{HbA1c}(\%)$ & 0.373 & 0.003 \\
\hline hs-CRP (mg/L) & 0.324 & 0.041 \\
\hline Total cholesterol (mg/dL) & 0.298 & 0.071 \\
\hline Triglycerides (mg/dL) & 0.254 & 0.052 \\
\hline HDL (mg/dL) & -0.145 & 0.320 \\
\hline LDL (mg/dL) & 0.221 & 0.089 \\
\hline eGFR-Cr $\left(\mathrm{mL} / \mathrm{min} / 1.73 \mathrm{~m}^{2}\right)$ & 0.105 & 0.415 \\
\hline ACR (mg/g creatinine) & 0.754 & 0.001 \\
\hline \multicolumn{3}{|c|}{$\begin{array}{l}\text { yrs: years, BMI: body mass index, SDS: standard deviation score, BP: blood pressure, LDL: low-density lipoprotein, HDL: high-density lipoprotein, ACR: urinary } \\
\text { albumin creatinine ratio, HbA1c: hemoglobin A1c, hs-CRP: high-sensitivity C-reactive protein, eGFR: estimated glomerular filtration rate }\end{array}$} \\
\hline \multicolumn{3}{|c|}{ Table 3. Multiple regression analysis of the relationship of midkine levels to clinical and laboratory variables } \\
\hline Variable & Standardized coefficients & $\mathrm{p}$ \\
\hline Age (yrs) & 0.24 & 0.48 \\
\hline Disease duration (yrs) & 0.38 & 0.09 \\
\hline SBP SDS & 0.22 & 0.86 \\
\hline DBP SDS & 0.54 & 0.08 \\
\hline hs-CRP (mg/L) & 0.61 & $<0.001$ \\
\hline $\mathrm{HbA1c}(\%)$ & 0.66 & $<0.001$ \\
\hline ACR (mg/g creatinine) & 0.49 & $<0.001$ \\
\hline
\end{tabular}

the studied groups was detected. Moreover, serum MK was not correlated with eGFR-Cr. These data indicate that serum MK is a better predictor of eGFR when compared to the classical eGFR-Cr method in children with T1DM, as eGFRCr was not able to detect the early renal effect in the present study.

In this study, HbA1c \% was significantly higher in Group 1 compared with Group 2 and Group 3. Moreover, there was also a significant correlation between HbA1c and MK. Kosugi et al (10) reported that MK expression was up-regulated by high glucose in primary cultured tubular epithelial cells. They also identify MK as a key molecule in patients with DN and suggested that MK accelerates the intracellular signaling network evoked by hyperglycemia in DN. High glucose levels and diabetic substrates, such as glycation end products, affect mostly proximal renal tubular cells, leading to tubular cell hypertrophy and the interstitial deposition of chemokines and cytokines like MK, which in turn cause inflammation and fibrosis of the tubules (31). In line with these data, Brito et al (32) have shown that the proximal tubular basement membrane is already thickened in normoalbuminuric patients with diabetes.

In the current study, we observed that the circulating levels of hs-CRP were significantly higher in Group 1 the other two groups and also in Group 2 compared with Group 3. Furthermore, the hsCRP levels were positively correlated with MK levels $(r=0.323, p=0.01)$. These findings support the hypothesis that MK, which is expressed in the proximal tubular epithelial cells of the kidney, plays a role in the pathophysiology of inflammation-related renal diseases (7). MK is an endogenous inflammatory marker and a key molecule in the development of DN. It enhances both neutrophil and macrophage migration into the tubulointerstitial regions, which is detrimental to kidney health (7). Studies involving human biopsy specimens and animal models have reported that macrophage infiltration is a characteristic of DN, confirming the concept that inflammation plays a crucial role in the pathogenesis of DN (33). 
In the current study, we showed that SBP SDS and DBP SDS were significantly higher in Group 1 compared to Group 2 and Group 3. Besides, DBP SDS was significantly higher in Group 2 than in Group 3. Hobo et al (34) showed that SBP and mean BP were significantly higher in the Mdk $+1+$ mice than in the Mdk - I - mice in the remnant kidney model as a model of advanced renal injury. They also reported that MK up-regulated pulmonary ACE in the 5/6 renal ablation model of CKD, leading to increase BP. These data suggest that MK plays an important role in the hypertensive response (29). MK was induced in the lung endothelium by oxidative stress and subsequently up-regulated by ACE, which hydrolyzes Ang II to induce more oxidative stress, thus accelerating MK generation. This leads to a vicious cycle of positive feedback in the MK-Ang II pathway (34). Kidney-lung interactions involving positive feedback between the renin-angiotensin system and MK might partly account for the pathogenesis of hypertension and kidney damage (35).

In our study, ROC curve analysis showed that an MK concentration cutoff value of $1512 \mathrm{pg} / \mathrm{mL}$ was able to predict microalbuminuria in children with T1DM with a sensitivity of $96 \%$ and specificity of $92 \%$. To the best of our knowledge, this is the first study to evaluate MK levels in children with DN and define a cut-off value. Therefore, further prospective studies are needed to validate this threshold.

\section{Study Limitations}

The small sample size is probably related to strict inclusion criteria of the studied cases.

Single-center study limits generalizability.

Due to the cross-sectional nature of the work, it is difficult to conclude whether higher MK levels are directly involved in the pathogenesis of DN complications or this is simply an association

We were also unable to compare the examined parameters based on a histological diagnosis. It should be taken into consideration that the increased levels of MK might not only reflect renal tubular cell damage. There may also be an extra-renal source.

Microalbuminuria was determined in spot urine samples rather than with a 24-hour urine sample, which is considered the standard method in determining microalbuminuria.

\section{Conclusion}

The results of this study suggest that serum MK is a useful, novel, practical marker for the evaluation of renal involvement in children with T1DM, especially in normoalbuminuric diabetic children. However, further research with a larger sample size and a prospective design are requied to clarify the predictive and pathophysiological role and significance of MK in the early phase and also in the progression of DN.

\section{Ethics}

Ethics Committee Approval: The study protocol was approved by the Ethics Committee of the Faculty of Medicine, Assiut Children University Hospital, Assiut, Egypt (approval number: 21/2017, date: 09.06.2021).

Informed Consent: Written informed consent was obtained from the parents of all participants.

Peer-review: Externally peer-reviewed.

\section{Authorship Contributions}

Surgical and Medical Practices: Kotb Abbass Metwalley, Hekma Saad Farghaly, Safwat Mohamed Abdel-Aziz, Asmaa Mohamed Ismail, Islam Fathy Elnakeeb, Concept: Kotb Abbass Metwalley, Hekma Saad Farghaly, Magda Farghali Gabri, Asmaa Mohamed Ismail, Duaa Mohamed Raafat, Islam Fathy Elnakeeb, Design: Kotb Abbass Metwalley, Hekma Saad Farghaly, Magda Farghali Gabri, Safwat Mohamed Abdel-Aziz, Islam Fathy Elnakeeb, Data Collection or Processing: Kotb Abbass Metwalley, Hekma Saad Farghaly, Asmaa Mohamed Ismail, Duaa Mohamed Raafat, Analysis or Interpretation: Kotb Abbass Metwalley, Hekma Saad Farghaly, Magda Farghali Gabri, Safwat Mohamed Abdel-Aziz, Asmaa Mohamed Ismail, Duaa Mohamed Raafat, Islam Fathy Elnakeeb, Literature Search: Kotb Abbass Metwalley, Hekma Saad Farghaly, Magda Farghali Gabri, Safwat Mohamed Abdel-Aziz, Duaa Mohamed Raafat, Asmaa Mohamed Ismail, Writing: Kotb Abbass Metwalley, Hekma Saad Farghaly.

Financial Disclosure: The authors declared that this study received no financial support.

\section{References}

1. Kadomatsu K, Kishida S, Tsubota S. The heparin-binding growth factor midkine: the biological activities and candidate receptors. J Biochem 2013;153:511-521

2. Weckbach LT, Groesser L, Borgolte J, Pagel JI, Pogoda F, Schymeinsky J, Müller-Höcker J, Shakibaei M, Muramatsu T, Deindl E, Walzog B. Midkine acts as proangiogenic cytokine in hypoxia-induced angiogenesis. Am J Physiol Heart Circ Physiol 2012;303:H429-H438. Epub 2012 Jun 15.

3. Kadomatsu K, Muramatsu T: Midkine and pleiotrophin in neural development and cancer. Cancer Lett 2004;204:127-143.

4. Weckbach LT, Muramatsu T, Walzog B. Midkine in inflammation. Scientific World Journal 2011;11:2491-2505.

5. Ikematsu S, Yano A, Aridome K, Kikuchi M, Kumai H, Nagano H, Okamoto K, Oda M, Sakuma S, Aikou T, Muramatsu H, Kadomatsu 
$\mathrm{K}$, Muramatsu T. Serum midkine levels are increased in patients with various types of carcinomas. Br J Cancer 2000;83:701-706.

6. Takada T, Toriyama K, Muramatsu H, Song XJ, Torii S, Muramatsu T. Midkine, a retinoic acid-inducible heparin-binding cytokine in inflammatory responses: chemotactic activity to neutrophils and association with inflammatory synovitis. J Biochem 1997;122:453458 .

7. Sato W, Kadomatsu K, Yuzawa Y, Muramatsu H, Hotta N, Matsuo S, Muramatsu T: Midkine is involved in neutrophil infiltration into the tubulointerstitium in ischemic renal injury. J Immunol 2001;167:34633469.

8. Kosugi T, Yuzawa Y, Sato W, Arata-Kawai H, Suzuki N, Kato N, Matsuo $\mathrm{S}$, Kadomatsu K. Midkine is involved in tubulointerstitial inflammation associated with diabetic nephropathy. Lab Invest 2007;87:903-913. Epub 2007 Jul 2.

9. Sato Y, Sato W, Maruyama S, Wilcox CS, Falck JR, Masuda T, Kadomatsu K. Midkine regulates BP through cytochrome P450-derived eicosanoids. J Am Soc Nephrol 2015;26:1806-1815.

10. Kosugi T, Yuzawa Y, Sato W, Kawai H, Matsuo S, Takei Y, Muramatsu T, Kadomatsu K. Growth factor midkine is involved in the pathogenesis of diabetic nephropathy. Am J Pathol 2006;168:9-19.

11. Kawai H, Sato W, Yuzawa Y, Kosugi T, Matsuo S, Takei Y, Kadomatsu $\mathrm{K}$, Muramatsu T. Lack of the growth factor midkine enhances survival against cisplatin-induced renal damage. Am J Pathol 2004;165:1603 1612 .

12. Fowler MJ. Microvascular and macrovascular complications of diabetes. Clin Diabet 2008;26:77-82

13. Pourghasem M, Shafi H, Babazadeh Z. Histological changes of kidney in diabetic nephropathy. Caspian J Intern Med 2015;6:120-127.

14. Haneda M, Utsunomiya K, Koya D, Babazono T, Moriya T, Makino H, Kimura K, Suzuki Y, Wada T, Ogawa S, Inaba M, Kanno Y, Shigematsu T, Masakane I, Tsuchiya K, Honda K, Ichikawa K, Shide K; Joint Committee on Diabetic Nephropathy. A new Classification of Diabetic Nephropathy 2014: a report from Joint Committee on Diabetic Nephropathy. J Diabetes Investig 2015;6:242-246. Epub 2015 Mar 1

15. Kaul A, Behera MR, Rai MK, Mishra P, Bhaduaria DS, Yadav S, Agarwal V, Karoli R, Prasad N, Gupta A, Sharma RK. Neutrophil gelatinaseassociated lipocalin: as a predictor of early diabetic nephropathy in type 2 diabetes mellitus. Indian J Nephrol 2018;28:53-60.

16. Tyagi I, Agrawal U, Amitabh V, Jain AK, Saxena S. Thickness of glomerular and tubular basement membranes in preclinical and clinical stages of diabetic nephropathy. Indian J Nephrol 2008;18:6469.

17. Gilbert RE, Cooper ME. The tubulointerstitium in progressive diabetic kidney disease: More than an aftermath of glomerular injury? Kidney Int 1999;56:1627-1637.

18. Kern EF, Erhard P, Sun W, Genuth S, Weiss MF. Early urinary markers of diabetic kidney disease: A nested case-control study from the Diabetes Control and Complications Trial (DCCT) Am J Kidney Dis 2010;55:824834
19. Pun KK, Ho P, Lau P, Wong FH. Eight-month longitudinal study of urinary excretion of albumin and tubular proteins in diabetic subjects. Am J Nephrol 1990;10:475-481.

20. Koroshi A. Microalbuminuria, is it so important? Hippokratia 2007;11:105-107.

21. Molitch ME, DeFronzo RA, Franz MJ, Keane WF, Mogensen CE, Parving HH, Steffes MW; American Diabetes Association. Nephropathy in diabetes. Diabetes Care 2004;27(Suppl 1):S79-S83.

22. Nielsen SE, Sugaya T, Tarnow L, Lajer M, Schjoedt KJ, Astrup AS, Baba T, Parving HH, Rossing P. Tubular and glomerular injury in diabetes and the impact of ACE inhibition. Diabetes Care 2009;32:1684-1688.

23. Diabetes Endocrine Metabolism Pediatric Unit, Cairo University Children's Hospital. Egyptian growth curves 2002. Last accessed date: 02.08.2021. Available from: http://dempuegypt.blogspot.com

24. Tanner JM. Growth at adolescence. Oxford: Blackwell Scientific Publications; 1962 .

25. National High Blood Pressure Education Program Working Group on High Blood Pressure in Children and Adolescents. The fourth report on the diagnosis, evaluation, and treatment of high blood pressure in children and adolescents. Pediatrics 2004;114(2 Suppl 4th Report):555-576.

26. Friedewald WT, Levy RI, Fredrickson DS. Estimation of the concentration of low-density lipoprotein cholesterol in plasma, without use of the preparative ultracentrifuge. Clin Chem 1972;18:499-502.

27. Schwartz GJ, Work DF. Measurement and estimation of GFR in children and adolescents. Clin J Am Soc Nephrol 2009;4:1832-1843. Epub 2009 Oct 9

28. Tonolo G, Cherchi S. Tubulointerstitial disease in diabetic nephropathy. Int J Nephrol Renovasc Dis 2014;7:107-115.

29. Sato W, Sato Y. Midkine in nephrogenesis, hypertension and kidney diseases. Br J Pharmacol. 2014;171:879-887.

30. American Diabetes Association. Standards of medical care in diabetes-Diabetes care. 2013;36(Suppl 1):S11-S66.

31. Tang SC, Lai KN. The pathogenic role of the renal proximal tubular cell in diabetic nephropathy. Nephrol Dial Transplant 2012;27:3049-3056.

32. Brito PL, Fioretto P, Drummond K, Kim Y, Steffes MW, Basgen JM, Sisson-Ross S, Mauer M. Proximal tubular basement membrane width in insulin-dependent diabetes mellitus. Kidney Int 1998;53:754-761.

33. Giralt-López A, Molina-Van den Bosch M, Vergara A, García-Carro C, Seron D, Jacobs-Cachá C, Soler MJ. Revisiting Experimental Models of Diabetic Nephropathy. Int J Mol Sci 2020;21:3587.

34. Hobo A, Yuzawa Y, Kosugi T, Kato N, Asai N, Sato W, Maruyama S, Ito Y, Kobori H, Ikematsu S, Nishiyama A, Matsuo S, Kadomatsu K. The growth factor midkine regulates the renin-angiotensin system in mice. J Clin Invest 2009;119:1616-1625. Epub 2009 May 18

35. Hoke TS, Douglas IS, Klein CL, He Z, Fang W, Thurman JM, Tao Y, Dursun B, Voelkel NF, Edelstein CL, Faubel S. Acute renal failure after bilateral nephrectomy is associated with cytokine-mediated pulmonary injury. J Am Soc Nephrol 2007;18:155-164. Epub 2006 Dec 13 